\title{
Uncharacterized RNAs in Plasma of Alzheimer's Patients Are Associated with Cognitive Impairment and Show a Potential Diagnostic Power
}

\author{
Cristina Barbagallo ${ }^{1}$, Maria Teresa Di Martino ${ }^{2}$ D , Margherita Grasso ${ }^{3,4}$, Maria Grazia Salluzzo ${ }^{3}$, \\ Francesca Scionti ${ }^{2}$, Filomena Irene Ilaria Cosentino ${ }^{3}$, Giuseppe Caruso ${ }^{3,4}$ (D), Davide Barbagallo ${ }^{1}$ (D), \\ Cinzia Di Pietro ${ }^{1} \mathbb{D}$, Raffaele Ferri ${ }^{3} \mathbb{D}$, Filippo Caraci ${ }^{3,4}{ }^{*} \mathbb{D}$, Michele Purrello ${ }^{1}$ and Marco Ragusa $^{1,3} \mathbf{D}^{\mathbb{D}}$ \\ 1 Department of Biomedical and Biotechnological Sciences, Section of Biology and Genetics G. Sichel, \\ University of Catania, 95123 Catania, Italy; barbagallocristina@unict.it (C.B.); dbarbaga@unict.it (D.B.); \\ dipietro@unict.it (C.D.P.); purrello@unict.it (M.P.); mragusa@unict.it (M.R.) \\ 2 Department of Experimental and Clinical Medicine, Magna Graecia University, 88100 Catanzaro, Italy; \\ teresadm@unicz.it (M.T.D.M.); fscionti20@gmail.com (F.S.) \\ 3 Oasi Research Institute-IRCCS, 94018 Troina, Italy; grassomargherita940@gmail.com (M.G.); \\ msalluzzo@oasi.en.it (M.G.S.); fcosentino@oasi.en.it (F.I.I.C.); forgiuseppecaruso@gmail.com (G.C.); \\ rferri@oasi.en.it (R.F.) \\ 4 Department of Drug Sciences, University of Catania, 95125 Catania, Italy \\ * Correspondence: carafil@hotmail.com
}

Received: 24 September 2020; Accepted: 13 October 2020; Published: 15 October 2020

\begin{abstract}
Alzheimer's disease (AD) diagnosis is actually based on clinical evaluation and brain-imaging tests, and it can often be confirmed only post-mortem. Therefore, new non-invasive molecular biomarkers are necessary to improve AD diagnosis. As circulating microRNA biomarkers have been proposed for many diseases, including AD, we aimed to identify new diagnostic non-small RNAs in AD. Whole transcriptome analysis was performed on plasma samples of five AD and five unaffected individuals (CTRL) using the Clariom D Pico Assay, followed by validation in real-time PCR on 37 AD patients and 37 CTRL. Six differentially expressed (DE) transcripts were identified: GS1-304P7.3 (upregulated), NONHSAT090268, TC0100011037, TC0400008478, TC1400008125, and UBE2V1 (downregulated). Peripheral blood mononuclear cells (PBMCs) may influence the expression of circulating RNAs and their analysis has been proposed to improve AD clinical management. Accordingly, DE transcript expression was also evaluated in PBMCs, showing no difference between AD and CTRL. ROC (receiver operating characteristic) curve analysis was performed to evaluate the diagnostic accuracy of each DE transcript and a signature including all of them. A correlation between cognitive impairment and GS1-304P7.3, NONHSAT090268, TC0100011037, and TC0400008478 was detected, suggesting a potential association between their extracellular abundance and AD clinical phenotype. Finally, this study identified six transcripts showing altered expression in the plasma of AD patients. Given the need for new, accurate blood biomarkers for AD diagnosis, these transcripts may be considered for further analyses in larger cohorts, also in combination with other biomarkers, aiming to identify specific RNA-based biomarkers to be eventually applied to clinical practice.
\end{abstract}

Keywords: AD; non-coding RNAs; lncRNAs; biomarkers; non-invasive diagnosis

\section{Introduction}

Alzheimer's disease (AD) is a neurodegenerative disorder and a leading cause of dementia in the elderly, accounting for $60-80 \%$ of all dementia cases worldwide. It is caused by the aggregation 
of misfolded proteins leading to the formation of extracellular amyloid plaques and intracellular neurofibrillary tangles of hyperphosphorylated tau protein [1]. To date, AD diagnosis is based on evaluation of cognitive symptoms (memory loss, disoriented behavior and impairments in language, comprehension, and spatial skills), combined with neuroimaging and neuropsychological markers [2]. Neuropsychiatric symptoms, such as depression, psychosis and agitation, are also frequent in people with $\mathrm{AD}$ and represent a common cause of hospitalization [3]. AD diagnosis can often be confirmed only though post-mortem analysis of brain tissues [4]. For this reason, research on appropriate diagnostic biomarkers is of paramount importance for a non-invasive diagnosis of AD and it has been very active since the early 2000s. The most important features for diagnostic biomarkers are not only sensitivity and specificity, making them able to discriminate affected from unaffected individuals with high accuracy, but also the low cost and the ease of sample collection and analysis. The most accurate biomarkers currently available for AD diagnosis are retrieved in cerebrospinal fluid (CSF), where it is possible to dose amyloid beta $(\mathrm{A} \beta)$, tau protein, and phosphorylated tau protein [5]. The great advantage of these biomarkers is their high sensitivity and specificity, likely due to the detection in CSF, which is in direct contact with the diseased brain. Unfortunately, the downside is that CSF collection is an invasive and expensive procedure. Therefore, researchers are actively working to identify new biomarkers that are easier to collect. The most common source of biomarkers is blood (specifically, serum, or plasma), as it can be collected easily and at low cost. Notably, blood-based biomarkers represent a novel tool to identify individuals at an early stage of AD [6]; indeed, in the last few decades, several blood circulating molecules have been investigated as AD diagnostic biomarkers, including $\mathrm{AD}$-related molecules ( $\mathrm{A} \beta$, tau and phosphorylated tau), proteins, metabolites, and microRNAs (miRNAs). However, several reports showed promising results that have not been confirmed in independent studies [7]. The application of circulating RNA molecules, including non-coding RNAs (ncRNAs), as biomarkers has been widely explored in the last few decades; miRNAs have been suggested as diagnostic biomarkers for several diseases, including neurodegenerative $[8,9]$, neurodevelopmental [10], traumatic [11], neoplastic [12,13], and metabolic conditions [14,15]. Indeed, miRNAs have been detected in all analyzed biological fluids [16-18], making them useful as disease biomarkers measurable in all body districts. Similarly, long non-coding RNAs (lncRNAs) and circular RNAs (circRNAs), a recently discovered class of ncRNAs, are emerging as new potential disease biomarkers, also in AD [19]. The exploitation of RNA molecules as diagnostic biomarkers would represent a great advantage for clinical practice: in fact, RNA is very easy to isolate and can be analyzed with high accuracy by common molecular biology techniques.

It has recently been proposed that immune system activation and neuroinflammation may also play an important role in AD pathogenesis [20]. Inflammatory processes have been observed in association with $\mathrm{AD}$, and a colocalization between $\mathrm{AD}$ lesions and pathogen signatures has been reported. Moreover, in vivo models showed that deposits of $A \beta$ and tau are induced by infections with both viruses and bacteria [21]. Given this association between $\mathrm{AD}$ pathogenesis and the immune system, several studies on immune cells in AD are emerging. Peripheral blood mononuclear cells (PBMCs) and lymphocytes have been proposed for their potential diagnostic value, because they are affected in AD patients and can reflect in the periphery biological alterations found in the central nervous system (CNS) [22,23]. Moreover, immune cells reside in blood and represent one of the most abundant cell types in this compartment of the body. Therefore, it may be hypothesized that differential expression of RNA-based biomarkers detected in blood could be influenced by expression levels of the transcripts detected in PBMCs.

Because of these reasons, the aim of this study was to identify new potential RNA-based biomarkers in plasma for $\mathrm{AD}$ diagnosis. Furthermore, we investigated the potential contribution of immune cells to the dysregulation of RNA molecules in the plasma of AD patients compared to unaffected individuals. 


\section{Results}

\subsection{Transcriptome Analysis of Plasma Samples of AD Patients Compared to Unaffected Individuals}

The whole transcriptome analysis performed using the Clariom D Pico assay investigated the expression of more than 540,000 transcripts and identified RNA molecules showing a differential expression between AD patients and unaffected individuals (CTRL). Microarray data were analyzed both considering the five AD-CTRL pairs and comparing the AD group with the CTRL group (paired and unpaired analyses, respectively). Results were filtered selecting only the transcripts showing a high fluorescence intensity in the microarray. We focused our attention on DE transcripts common to paired and unpaired results, filtered according to $p$-value and fold change, obtaining a total of 325 deregulated transcripts, of which 215 were upregulated and 110 were downregulated (Figure 1).

A

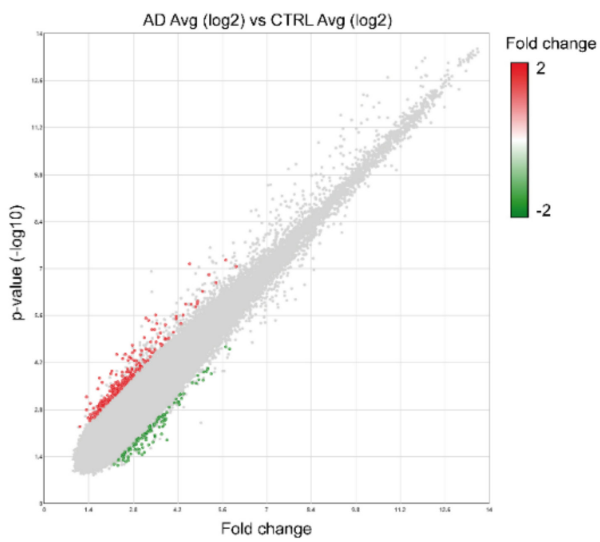

C

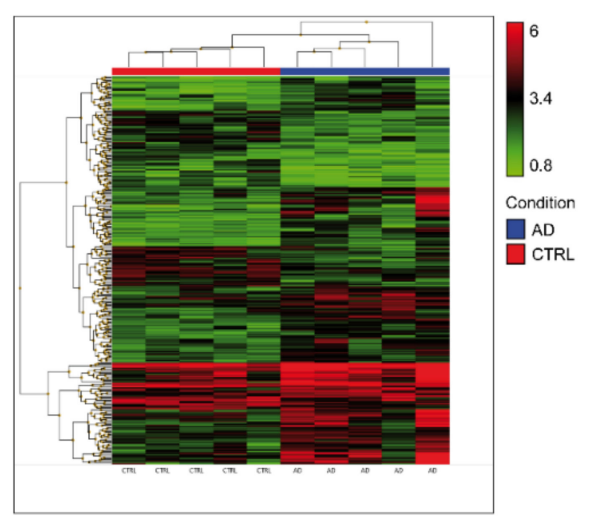

B

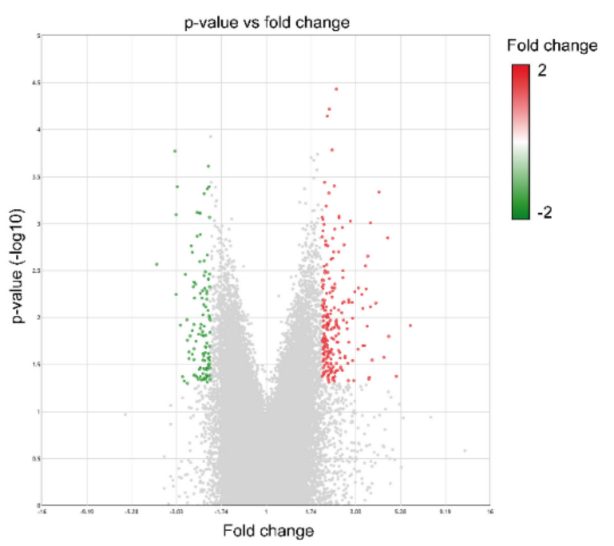

Figure 1. Results of microarray profiling. (A) scatter plot showing fluorescence intensity of significantly deregulated transcripts; (B) volcano plot showing significantly deregulated transcripts; (C) hierarchical clustering of analyzed samples. Legend: (A) and (B) colored dots show significantly deregulated transcripts $(p<0.05)$, with red representing upregulation (fold change $>2$ ) and green representing downregulation (fold change $<-2$ ); (C) fluorescence intensity data are plotted. AD: Alzheimer's disease patients; CTRL: unaffected individuals.

\subsection{Validation of Microarray Results by Real-Time PCR}

DE transcripts identified through microarray profiling were successively validated in an independent cohort of 74 individuals, including 37 AD patients and 37 unaffected controls, matched for sex and age. We selected 48 transcripts from profiling results, showing the highest fluorescence intensity and the strongest $p$-values and fold changes. First of all, the expression of these transcripts was evaluated in a small cohort of plasma samples (data not shown) to assess their amplificability in PCR; 
accordingly, validation analysis was performed on 19 transcripts showing detectable levels in Real-Time PCR. Validation assays confirmed the differential expression of 6 transcripts, namely GS1-304P7.3, NONHSAT090268, TC0100011037, TC0400008478, TC1400008125, and UBE2V1. For all transcripts, both paired and unpaired analyses showed statistical significance according to both endogenous controls. In particular, GS1-304P7.3 showed increased expression in the AD group compared to the CTRL group, while NONHSAT090268, TC0100011037, TC0400008478, TC1400008125, and UBE2V1 levels were reduced in AD plasma samples compared to the CTRL group (Table 1).

Table 1. Real-Time PCR results. For each transcript, TAC ID, gene symbol (where available), median fold change and $p$-value (between brackets) are shown for each endogenous control (GAPDH and RNU6) and for both paired and unpaired analyses. Significant values are highlighted in bold. TAC: Transcriptome Analysis Console; GAPDH: glyceraldehyde-3-phosphate dehydrogenase; RNU6: RNA, U6 small nuclear 1.

\begin{tabular}{|c|c|c|c|c|c|}
\hline \multirow{2}{*}{ TAC ID } & \multirow{2}{*}{ Gene Symbol } & \multicolumn{2}{|c|}{ GAPDH } & \multicolumn{2}{|c|}{ RNU6 } \\
\hline & & Paired & Unpaired & Paired & Unpaired \\
\hline TC0100010930 & GS1-304P7.3 & $1.93(0.027)$ & $2.79(0.008)$ & $1.91(0.043)$ & $2.18(0.04)$ \\
\hline TC0100011037 & & $-2.16(0.045)$ & $-2.58(0.046)$ & $-3.17(0.006)$ & $-3.21(0.01)$ \\
\hline TC0100013007 & & $-1.12(0.63)$ & $-1.21(0.44)$ & $1.09(0.77)$ & $1.04(0.5)$ \\
\hline TC0100015528 & & $1.18(0.86)$ & $1.24(0.87)$ & $2.48(0.84)$ & $2(0.83)$ \\
\hline TC0100016418 & & $-1.31(0.52)$ & $-1.31(0.51)$ & $-1.52(0.64)$ & $-2.07(0.64)$ \\
\hline TC0300007694 & NONHSAT090268 & $-2.74(0.007)$ & $-3.04(0.006)$ & $-1.71(0.007)$ & $-3.74(0.008)$ \\
\hline TC0300013071 & zyjeebu & $-1.78(0.71)$ & $-2.36(0.65)$ & $-1.77(0.83)$ & $-1.44(0.82)$ \\
\hline TC0400008478 & & $-15.75(0.016)$ & $-11.22(0.003)$ & $-63.17(0.016)$ & $-7.04(0.002)$ \\
\hline TC0500012139 & peybleeby & $-1.22(0.8)$ & $1.17(0.74)$ & $-1.33(0.68)$ & $1.12(0.9)$ \\
\hline TC0600007285 & HIST1H2AE & $1.06(0.98)$ & $1.57(0.98)$ & $1.36(0.48)$ & $-1.78(0.47)$ \\
\hline TC0600007784 & & $-1.23(0.99)$ & $-1.23(0.32)$ & $-1.31(0.79)$ & $1.48(0.81)$ \\
\hline TC0800009993 & blawker & $-1.15(0.91)$ & $2.43(0.88)$ & $1.47(0.72)$ & $1.76(0.66)$ \\
\hline TC1000010059 & NONHSAT011783 & $1.19(0.37)$ & $1.85(0.19)$ & $1.3(0.88)$ & $-1.35(0.91)$ \\
\hline TC1200011311 & NAP1L1 & $1.07(0.99)$ & $4.21(0.85)$ & $1.17(0.9)$ & $3.9(0.85)$ \\
\hline TC1400008125 & & $-1.55(0.037)$ & $-2.25(0.032)$ & $-1.62(0.021)$ & $-2.36(0.042)$ \\
\hline TC1600007870 & & $1.08(0.99)$ & $-2.04(0.99)$ & $-1.35(0.95)$ & $1.02(0.94)$ \\
\hline TC1600010293 & swoyry & $-1.27(0.3)$ & $-1.76(0.5)$ & $-1.78(0.06)$ & $-3.32(0.09)$ \\
\hline TC1900010363 & & $-2.57(0.67)$ & $-2.46(0.71)$ & $-1.59(0.63)$ & $1.02(0.56)$ \\
\hline TC2000010025 & UBE2V1 & $-1.37(0.037)$ & $-1.63(0.048)$ & $-1.98(0.028)$ & $-2.1(0.045)$ \\
\hline
\end{tabular}

Statistical analyses performed to evaluate age and sex as confounding factors showed no significant results, confirming that these parameters do not represent confounding factors in our study (data not shown).

We also investigated the existence of any difference in DE transcript expression associated with gender. This analysis was performed within each pathological group (AD males vs females and CTRL males vs. females) and within individuals of the same gender (female AD vs CTRL and male AD vs CTRL): no statistical difference was observed.

\subsection{Evaluation of Diagnostic Accuracy through ROC Curves}

The identification of differentially expressed (DE) transcripts in plasma of AD patients compared to unaffected individuals may pave the way to potential diagnostic applications of these transcripts in clinical practice. For this reason, we computed receiver operating characteristic (ROC) curves to assess the potential diagnostic accuracy of DE transcripts. First of all, we evaluated the accuracy of each DE transcript, considered as an individual plasma biomarker for AD. Univariable ROC curves are shown in Table 2 and Figure 2. 
Table 2. Results of univariable ROC (receiver operating characteristic) curve analysis. For each transcript, the $p$-value of the curve, the area under the curve (AUC), its standard error (Std error), and the $95 \%$ confidence intervals (CIs) are shown; the Youden index was also calculated to identify the optimal cut-off, for which sensitivity, specificity, accuracy, positive predictive value (PPV) and negative predictive value (NPV) are shown. Significant values are highlighted in bold.

\begin{tabular}{ccccccccccc}
\hline DE Transcript & AUC & Std Error & $\boldsymbol{P}$-Value & $\mathbf{9 5 \%}$ CIs & Cut-Off & Sensitivity & Specificity & Accuracy & PPV & NPV \\
\hline GS1-304P7.3 & $\mathbf{0 . 7 2 2}$ & $\mathbf{0 . 0 7 4}$ & $\mathbf{0 . 0 0 8}$ & $\mathbf{0 . 5 7 8 - 0 . 8 6 7}$ & $\mathbf{1 . 8 5}$ & $\mathbf{0 . 7 5}$ & $\mathbf{0 . 7 1}$ & $\mathbf{0 . 7 3}$ & $\mathbf{0 . 7 2}$ & $\mathbf{0 . 7 4}$ \\
NONHSAT090268 & $\mathbf{0 . 7 5 3}$ & $\mathbf{0 . 0 6 9}$ & $\mathbf{0 . 0 0 2}$ & $\mathbf{0 . 6 1 8 - 0 . 8 8 7}$ & $\mathbf{3 . 1 2}$ & $\mathbf{0 . 6 8}$ & $\mathbf{0 . 8 1}$ & $\mathbf{0 . 7 4}$ & $\mathbf{0 . 7 7}$ & $\mathbf{0 . 7 1}$ \\
TC0100011037 & $\mathbf{0 . 7 1 6}$ & $\mathbf{0 . 0 7 2}$ & $\mathbf{0 . 0 0 6}$ & $\mathbf{0 . 5 7 6 - 0 . 8 6 5}$ & $\mathbf{2 . 6 6}$ & $\mathbf{0 . 6 7}$ & $\mathbf{0 . 7 4}$ & $\mathbf{0 . 7}$ & $\mathbf{0 . 7 2}$ & $\mathbf{0 . 6 9}$ \\
TC0400008478 & $\mathbf{0 . 8 0 3}$ & $\mathbf{0 . 0 7 8}$ & $\mathbf{0 . 0 0 1}$ & $\mathbf{0 . 6 5 - 0 . 9 5 7}$ & $\mathbf{1 . 9 7}$ & $\mathbf{0 . 9 5}$ & $\mathbf{0 . 6 8}$ & $\mathbf{0 . 8 2}$ & $\mathbf{0 . 7 5}$ & $\mathbf{0 . 9 3}$ \\
TC1400008125 & 0.644 & 0.076 & 0.064 & $0.494-0.794$ & 2.07 & 0.5 & 0.89 & 0.7 & 0.82 & 0.64 \\
UBE2V1 & $\mathbf{0 . 6 3 7}$ & $\mathbf{0 . 0 6 5}$ & $\mathbf{0 . 0 4 5}$ & $\mathbf{0 . 5 1 - 0 . 7 6 5}$ & $\mathbf{1 . 9 5}$ & $\mathbf{0 . 7 8}$ & $\mathbf{0 . 4 7}$ & $\mathbf{0 . 6 3}$ & $\mathbf{0 . 6}$ & $\mathbf{0 . 6 8}$ \\
\hline
\end{tabular}

\section{ROC curves}

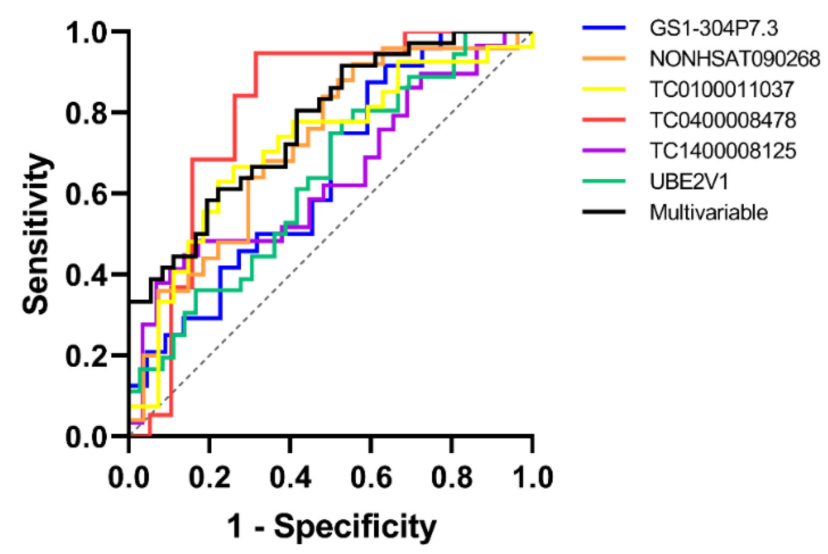

Figure 2. Univariable and multivariable ROC curves computed on plasma expression levels of differentially expressed (DE) transcripts.

Results showed significant curves for GS1-304P7.3, NONHSAT090268, TC0100011037, TC0400008478, and UBE2V1, with a good diagnostic performance for TC0400008478 (AUC > 0.8) and a fair performance (AUC > 0.7) for GS1-304P7.3, NONHSAT090268, and TC0100011037.

Moreover, we evaluated the diagnostic accuracy of a biomarker signature including all six DE transcripts; this multivariable ROC curve showed a significant $p$-value but did not improve the diagnostic performances of single univariate univariable ROC curves (Table 3 and Figure 2).

Table 3. Features of the ROC curve computed on all DE transcripts. The $p$-value of the curve, the AUC, its standard error (Std error), and the 95\% CIs are shown; sensitivity, specificity, accuracy, PPV and NPV are also shown. Significant values are highlighted in bold.

\begin{tabular}{cccccccccc}
\hline Transcript Signature & $P$-Value & AUC & $\begin{array}{c}\text { Std } \\
\text { Error }\end{array}$ & $\begin{array}{c}95 \% \\
\text { CIs }\end{array}$ & Sensitivity & Specificity Accuracy & PPV & NPV \\
\hline $\begin{array}{c}\text { GS1-304P7.3, } \\
\text { NONHSAT090268, }\end{array}$ & 0.00007 & 0.772 & 0.054 & $0.667-0.878$ & 0.64 & 0.72 & 0.68 & 0.7 & 0.67 \\
$\begin{array}{c}\text { TC0100011037, TC0400008478, } \\
\text { TC1400008125, UBE2V1 }\end{array}$ & & & & & & & & & \\
\hline
\end{tabular}

\subsection{Peripheral Blood-Isolated Cell Expression Analysis}

Expression of DE transcripts was also evaluated in PBMCs obtained from AD patients and age-and sex-matched unaffected individuals. Although real-time PCR results showed an expression trend similar to that reported in plasma (except for GS1-304P7.3), no statistically significant difference was observed (Table 4). 
Table 4. Expression analysis performed on PBMCs. For each transcript, median fold change and $p$-value (between brackets) are shown for each endogenous control (ACTB and RNU6). ACTB: actin beta.

\begin{tabular}{ccc}
\hline Transcript & ACTB & RNU6 \\
\hline GS1-304P7.3 & $-2.55(0.37)$ & $-1.42(0.51)$ \\
NONHSAT090268 & $-2.51(0.36)$ & $-1.41(0.36)$ \\
TC0100011037 & $-2.54(0.37)$ & $-1.42(0.51)$ \\
TC0400008478 & $-2.49(0.39)$ & $-1.39(0.54)$ \\
TC1400008125 & $-3.01(0.39)$ & $-1.68(0.34)$ \\
UBE2V1 & $-1.52(0.53)$ & $1.17(0.47)$ \\
\hline
\end{tabular}

\subsection{Correlation with Clinical and Cognitive Phenotypes}

Plasma levels of DE transcripts and clinicopathological parameters measuring cognitive impairment and its progression were correlated. A significant correlation between Mini Mental State Examination (MMSE) T0 and four out of the six DE transcripts was observed: in particular, a negative correlation was observed between MMSE score at T0 and GS1-304P7.3, the only transcript showing increased expression in AD patients, while NONHSAT090268, TC0100011037, and TC0400008478, downregulated in the plasma of AD patients, showed a positive correlation with MMSE T0. No significant correlation was observed between Delta MMSE/month and any transcripts (Table 5).

Table 5. Correlation analysis between plasma levels of DE transcripts and clinicopathological parameters. For each transcript/parameter pair, the $r$-value (Pearson or Spearman correlation coefficient, according to normality of distributions) and the $p$-value corrected for multiple comparison (between brackets) are shown. Significant values are highlighted in bold. MMSE: Mini Mental State Examination.

\begin{tabular}{ccc}
\hline DE Transcript & MMSE T0 & Delta MMSE/Month \\
\hline GS1-304P7.3 & $\mathbf{- 0 . 3 6 ( \mathbf { 0 . 0 2 4 } )}$ & $0.29(0.17)$ \\
NONHSAT090268 & $\mathbf{0 . 3 8}(\mathbf{0 . 0 1 2})$ & $0.14(0.51)$ \\
TC0100011037 & $\mathbf{0 . 3 2}(\mathbf{0 . 0 3 )}$ & $0.16(0.42)$ \\
TC0400008478 & $\mathbf{0 . 4 8 ( \mathbf { 0 . 0 2 } )}$ & $0.33(0.13)$ \\
TC1400008125 & $0.02(0.85)$ & $-0.1(0.85)$ \\
UBE2V1 & $-0.05(0.67)$ & $-0.14(0.65)$ \\
\hline
\end{tabular}

\section{Discussion}

Blood-based biomarkers will become essential in the near future to perform non-invasive diagnosis of AD. According to this scenario, RNA-based diagnostic biomarkers may represent a novel tool to improve the diagnosis of AD. The aim of this study was to perform a transcriptome analysis of plasma from mild to moderate AD patients and unaffected individuals, in order to identify RNA transcripts (including mRNAs, IncRNAs and circRNAs) to be applied as new potential diagnostic biomarkers. Our cohort included AD patients and unaffected individuals matched for sex and age, in order to exclude the influence of these features on the transcriptome. Through microarray analysis and subsequent validation in real-time PCR, we identified six transcripts showing differential expression; in particular, GS1-304P7.3 was upregulated in the AD group compared to the CTRL group, while NONHSAT090268, TC0100011037, TC0400008478, TC1400008125, and UBE2V1 showed reduced expression in $\mathrm{AD}$ plasma samples compared to the CTRL group. The same expression alteration was confirmed by both paired and unpaired analyses, showing that sex and age did not influence transcript expression in our cohort, suggesting the potential strength of these biomarkers. It has recently been hypothesized that immune system activation plays a key role in AD pathogenesis [20]. The key contribution of inflammation in AD pathology was hypothesized more than twenty years ago and recent studies have demonstrated that this early disease-aggravating CNS inflammation starts decades before the presentation of severe cognitive impairments or $\mathrm{AD}$ [24]. The potential role of the immune system cells may be enhanced by their abundant presence in blood, where they represent one of the 
principal cell types: indeed, altered expression of ncRNAs in immune cells may influence expression profiles of the same ncRNAs circulating in the blood. For these reasons, we also investigated the expression of these transcripts in PBMCs isolated from a sub-cohort of AD patients and unaffected individuals. However, this analysis showed no significant differential expression within PBMCs. Further studies are needed in larger cohorts of AD patients.

Among the transcripts identified here as differentially expressed in the plasma of AD patients compared with unaffected individuals, $U B E 2 V 1$ is the only protein-coding and characterized one. UBE2V1 (ubiquitin conjugating enzyme E2 V1) encodes a ubiquitin-conjugating enzyme involved in the proteasome degradation pathway. A recent study showed that $U B E 2 V 1$ promotes protein aggregation in rat cardiomyocytes [25]; this report could be relevant for a potential involvement of $U B E 2 V 1$ in $A D$, where protein aggregation and amyloid deposition is one of the histopathological hallmarks. Moreover, reduced UBE2V1 levels were observed in the cerebellum and blood of a mouse model of Rett syndrome, a rare neuropsychiatric disorder [26]. No data are available to date on humans, but reports in animal models suggest that $U B E 2 V 1$ plays a key role in synaptic plasticity and function by affecting postsynaptic density-95 (PSD95) scaffolding properties [27]. We found a reduction of $U B E 2 V 1$ in the plasma of AD patients. This result would suggest that $U B E 2 V 1$ might be analyzed in the near future as a novel pharmacological target in AD. The other DE transcripts are uncharacterized, and very scarce data are available in the literature. By blasting sequence transcripts, we investigated their position within the genome, aiming to infer some information about their putative functions. These transcripts could act as regulators of expression of the neighbor genes by binding regulatory sequences, such as silencers/enhancers [28], or as splicing regulators of host genes when their sequences overlap [29]. Therefore, by mapping DE transcripts on the genome, we could speculate on their indirect involvement in $\mathrm{AD}$ pathogenesis, performed by regulating the expression of neighbor genes involved in the disease. GS1-304P7.3 (TC0100010930) maps on chromosome 1, in an intergenic region between the lncRNA PDC-AS1 (about $600 \mathrm{bp}$ upstream) and the locus of PTGS2 (about $149 \mathrm{~Kb}$ downstream). GS1-304P7.3 is known to be expressed in endothelial cells, suggesting its involvement in angiogenetic processes [30]. A link between $\mathrm{AD}$ and angiogenesis was previously reported, showing a direct involvement of endothelial cells in the neovascularization process occurring in AD brain [31,32]. No information is available on PDC-AS1 (PDC antisense RNA 1), but the function of its sense transcript $P D C$ (phosducin) has been described. The exact function of antisense transcripts has not been elucidated, but evidence suggests the existence of a mutual post-transcriptional regulation involving sense and antisense transcripts [33]. PDC, encoding a phosphoprotein involved in the process of vision in the retina, has been associated with stress-induced hypertension [34,35]. Hypertension, in turn, has been associated with an increased risk to develop AD [36,37]. PTGS2 (prostaglandin-endoperoxide synthase 2) encodes the cyclooxygenase enzyme, involved in the biosynthesis of prostaglandins; different single nucleotide polymorphisms in PTGS2 have been associated with $\mathrm{AD}$, resulting in protection or increased risk of developing the disease [38,39]. In vivo experiments in mouse models showed that inhibition of PTGS2 expression is increased by A $\beta$ deposition, leading to inflammation [40], while inhibition of the enzyme prevents memory deficits [41]. TC0100011037 maps on an intergenic region in chromosome 1 , between the IncRNA LINC01031 (about $100 \mathrm{~kb}$ upstream) and the pseudogene RPL23AP22 (about $286 \mathrm{~kb}$ downstream). No information is available in the literature on both the neighbor genes. However, $R P L 23 A P 22$ is a pseudogene of RPL23A (ribosomal protein L23a), a ribosomal protein that has been associated with AD pathogenesis in transgenic models [42,43]. TC0400008478 maps on chromosome 4, between the genetic loci of ARSJ (about $269 \mathrm{~kb}$ upstream) and UGT8 (about $344 \mathrm{~kb}$ downstream). ARSJ (arylsulfatase family member J) encodes a sulfatase enzyme that has not been associated with AD to date. UGT8 (UDP glycosyltransferase 8) encodes a UDP-glycosyltransferase involved in the biosynthesis of galactocerebrosides, sphingolipids abundant in the central nervous system [44]. UGT8 has been reported as a key gene in a mouse model of $\mathrm{AD}$, where its genetic ablation induced the dysregulation of the same myelination pathways altered in human AD brain [45]. Moreover, UGT8 expression was shown to increase with cognitive impairment in human brain [46]. TC1400008125 
maps on chromosome 14, within a 37-kb intron of the lncRNA LINC02299. No information is available on the function of this lncRNA. NONHSAT090268 (TC0300007694) maps on chromosome 3, within a 141-kb intron of the lncRNA ADAMTS9-AS2. ADAMTS9-AS2 (ADAMTS9 antisense RNA 2) is an antisense lncRNA with unknown function, recently reported as a tumor suppressor gene in glioma [47] and associated with senile neurodegeneration and AD [48]. Its sense transcript, ADAMTS9 (ADAM metallopeptidase with thrombospondin type 1 motif 9), encodes for a disintegrin and metalloproteinase with thrombospondin motifs enzyme; single nucleotide polymorphisms in ADAMTS9 sequence have been associated with cognitive aging [49]. ADAMTS9 suppresses $\beta$-catenin and the canonical Wnt signaling pathway [50] that is known to be inhibited in the AD brain [51,52].

Hypothesizing a potential application as diagnostic plasma biomarkers for these transcripts, we evaluated their diagnostic performance by computing ROC curves. We computed two types of ROC curves, aiming to identify the best biomarker or combination of biomarkers, creating a signature specific for $\mathrm{AD}$ diagnosis. It is now widely proven that a signature including multiple biomarkers may show higher sensitivity and specificity than a single one. Univariable ROC curves were computed considering each biomarker by itself in order to test the diagnostic accuracy of the single transcript. We obtained significant curves for GS1-304P7.3, NONHSAT090268, TC0100011037, TC0400008478, and UBE2V1, but only the curve built on TC0400008478 reached a good diagnostic performance (AUC > 0.8). Similarly, a signature including all six biomarkers did not increase diagnostic performance. To date, the best diagnostic performance for AD has been reported for biomarkers detected in CSF [53]. This observation is not surprising, since CSF is in direct contact with brain interstitial fluid; therefore, biomarkers in CSF are specific of the brain and not diluted by others originating from different body districts (two conditions that characterize biomarkers detected in blood). Unfortunately, CSF sampling is invasive for patients and economically expensive, making blood-based biomarkers, even with their disadvantages, more attractive. According to their dilution in the circulation, blood biomarkers showed a lower diagnostic performance in different reports: a recent study analyzed the diagnostic accuracy of total $A \beta 42$-to- $A \beta 40$ ratio and free $A \beta 42$-to- $A \beta 40$ ratio in plasma, showing AUC values of 0.775 and 0.710, respectively [54]; another study reported the diagnostic performances of $A \beta 1-42$, and of the ratios of $A \beta 1-42$ to a novel APP669-711 fragment (APP669-711/A $\beta 1-42$ ) and A $\beta 1-40 / A \beta 1-42$, analyzed in different cohorts of patients, resulting in ROC curves with variable AUCs [55]. Diagnostic performance of DE transcripts here identified conforms with $A \beta$-based blood biomarkers currently reported in the literature. However, it is important to underline that the diagnostic power reported for a specific biomarker is very difficult to validate in a different study. This difficulty could be explained by the diversity of the enrolled cohort (age, ethnicity, diagnostic criteria), the different procedure and technique applied for biomarker detection and measurement, the different lab equipment used. Therefore, the best and only way to compare diagnostic accuracy of DE transcripts and previously proposed biomarkers would be to perform a dedicated study, where all different biomarkers are measured in the same patients with the same workflow and technique. Looking for the best diagnostic performance, it may be useful to combine RNA-based (including both small and long non-coding RNAs) and A $\beta$-based biomarkers from plasma to assess the diagnostic accuracy of an AD specific signature including both types of biomarkers. Further studies are needed to investigate the efficacy of such signatures in large and multicentric cohorts of patients.

Correlation analysis showed that four out of the six DE transcript are associated with cognitive impairment. In particular, GS1-304P7.3 showed a negative correlation with MMSE score, while NONHSAT090268, TC0100011037, and TC0400008478 showed a positive correlation with global cognitive function. These opposite correlation trends are in agreement with the opposite deregulation trends observed in plasma, where GS1-304P7.3 was the only transcript with increased levels in AD patients, while the other RNA-based biomarkers showed a reduced expression. This association with cognitive impairment suggests an active role of these transcripts in AD pathogenic processes that might be better examined in animal models of AD. 


\section{Materials and Methods}

\subsection{Patient Recruitment and Plasma Sample Processing}

This study was approved by the Ethics Committee of Oasi Research Institute-IRCCS, Troina (Italy) (20 March 2019; Code: 2019/03/18/CE-IRCCS-OASI/18) and it was performed in accordance with the Declaration of Helsinki. A total of 84 participants, consisting of 42 AD mild-moderate patients and 42 CTRL, were included in this study. AD patients were recruited at the Oasi Research Institute-IRCCS, Troina (Italy) (Table 6), where the NINCDS-ADRDA criteria (1984) for AD was used [56] in combination with DSM-IV guidelines. Written informed consent was obtained from all the participants or their families. Unaffected individuals matched for age and sex, with no cognitive impairment and family history of AD or Vascular Dementia, were selected. Total cognitive function assessment was carried out by using the Mini-Mental State Examination (MMSE) test [57]. For AD patients, total cognitive function was measured at both baseline (T0) and after 6-24 months (T1). Delta MMSE/month, which represents the progression rate of cognitive decline [58], was calculated as follows:

(MMSE score at T1-MMSE score at T0)/Interval between follow-up visit (months)

Table 6. Clinicopathological features of AD patients and unaffected individuals enrolled for this study. Data are presented as average \pm standard deviation. AD: Alzheimer's disease; N/A: not available.

\begin{tabular}{cccccc}
\hline & Sex (M/F) & Age & MMSE T0 & MMSE T1 & Delta MMSE/Month \\
\hline AD & $17 / 25$ & $74.51 \pm 6.95$ & $18.58 \pm 5.4$ & $14.54 \pm 6.01$ & $-0.32 \pm 0.21$ \\
CTRL & $17 / 25$ & $73.72 \pm 7.34$ & $29.64 \pm 0.48$ & N/A & N/A \\
\hline
\end{tabular}

For plasma isolation, blood samples were collected in vacutainer tubes and centrifuged at $1800 \times g$ for $15 \mathrm{~min}$ at $20^{\circ} \mathrm{C}$; plasma was stored at $-80^{\circ} \mathrm{C}$ until analysis.

\subsection{RNA Isolation from Plasma Samples}

Total RNA was isolated from plasma samples using the miRNeasy Mini-Kit (Qiagen, Hilden, Germany), according to the Qiagen supplementary protocol for total RNA isolation from serum and plasma. RNA was finally eluted in $200 \mu \mathrm{L}$ RNase-free water and then precipitated by adding $3 \mathrm{M}$ sodium acetate ( $\mathrm{pH} 5.2$ ), 100\% ethanol, and $1 \mu \mathrm{g}$ glycogen as carrier. After overnight incubation at $-80{ }^{\circ} \mathrm{C}$, samples were centrifuged $\left(12,000 \times g, 30 \mathrm{~min}, 4^{\circ} \mathrm{C}\right)$ and pellets were washed twice in 75\% ethanol. The final RNA pellet was dissolved in RNase-free water. RNA quantification was performed by Nanodrop One (Thermo Fisher Scientific, Waltham, MA, USA).

\subsection{Microarray Analysis}

Whole transcriptome analysis of plasma was performed in $5 \mathrm{AD}$ patients and 5 matched controls by using Clariom D Pico Assay (Thermo Fisher Scientific, Waltham, MA, USA). This technology analyzes the expression of more than 540,000 coding and non-coding transcripts, including mRNAs, circRNAs, lncRNAs, miRNA precursors and other small RNAs, loading a low input of total RNA. Briefly, $10 \mathrm{ng}$ of total RNA were retrotranscribed in single-stranded cDNA containing T7 promoter sequence at the $5^{\prime}$ end. $3^{\prime}$ Double-stranded cDNA was synthesized by adding an adaptor as a template; pre-IVT amplification reaction was optimized with 12 cycles of amplification, as previously reported [59]. The double-stranded DNA was used as a template for antisense RNA synthesis and overnight amplification (14 h) by in vitro transcription (IVT), using T7 RNA polymerase. Approximately $20 \mu \mathrm{g}$ of purified cRNA were used for sense single-strand cDNA (ss-cDNA) synthesis, followed by RNase $\mathrm{H}$ digestion and ss-cDNA magnetic bead purification. Approximately $5.5 \mu \mathrm{g}$ of ss-cDNA were fragmented using uracil DNA-glycosylase $(10 \mathrm{U} / \mu \mathrm{L})$ and apurinic/apyrimidinic endonuclease $1(1.000 \mathrm{U} / \mu \mathrm{L})$, and then labeled with biotin using terminal deoxynucleotidyl transferase $(30 \mathrm{U} / \mu \mathrm{L})$. 
From the hybridization cocktail, $200 \mu \mathrm{L}$ of the obtained mixture were loaded into single human Clariom D 49-format array and incubated for $16 \mathrm{~h}$ in the Affymetrix GeneChip Hybridization Oven 645 at $45^{\circ} \mathrm{C}, 60 \mathrm{rpm}$. Arrays were stained using an Affymetrix GeneChip Fluidics Station 450, according to the specific fluidics protocol (FS450_0001), and scanned with an Affymetrix GeneChip Scanner 3000 7G. Raw intensity CEL files generated by GeneChip ${ }^{\mathrm{TM}}$ Command Console ${ }^{\mathrm{TM}}$ were imported into Transcriptome Analysis Console (TAC) 4.0 (Applied Biosystems) and CHP files were generated for gene-level analysis. Differentially expressed (DE) transcripts were identified by using TAC, with the following settings: Analysis Type: Expression Gene; Summarization Method: Gene Level - RMA. Gene-Level $P$-Value $<0.05$ ANOVA Method: ebayes. Simultaneously, microarray data were also analyzed by MeV (Multi Experiment Viewer) v4.9.0 (http://mev.www.tm4.org, accessed on 19 July 2018) by applying Significance of Microarrays Analysis (SAM); paired and unpaired tests were performed among $\Delta C$ ts using a $p$-value based on 100 permutations; imputation engine: K-nearest neighbors (10 neighbors); false discovery rate (FDR) $<0.05$. Results from paired and unpaired analyses were compared, selecting common transcripts for validation analysis.

\subsection{Validation of Microarray Results Using Real-Time PCR}

DE transcripts identified in the microarray analysis were validated in an independent cohort of 37 AD patients and 37 age- and sex-matched controls. PCR primers for the DE transcripts were designed using PrimerBlast (https://www.ncbi.nlm.nih.gov/tools/primer-blast/, accessed on 10 December 2018) (Table 7).

Table 7. PCR primers used for Real-Time PCR validation assays.

\begin{tabular}{ccc}
\hline Transcript & Forward Primer & Reverse Primer \\
\hline ACTB & GAGCACAGAGCCTCGCCTTT & GAGCGCGGCGATATCATCA \\
blawker & AACCTGGGGCTGGTAAAGGTA & TGTGCTGCTGTTTGGTAGTCA \\
GS1-304P7.3 & TGCACCACCAACTGCTTAGC & GGCATGGACTGTGGTCATGAG \\
HIST1H2AE & AAGAAGACCAGAACAGATAGT & GGTCCTAGACACTGACGAAATC \\
NAP1L1 & GGCAGACATTGACAACAAAGAAC & GACTCGGGATCACTGACGGA \\
NONHSAT011783 & TTGGTGATAGAAAAGGGCTGAAGT & GTGGCTCTCTCGGACAATGC \\
NONHSAT090268 & TCTGGCCTTACCACCTCCTTT & GAGTGGAAATGACAACTTGATGCTC \\
peybleeby & ATGGTACAGGGTGATGGGCT & GCACCCTCCCCCACCTAATA \\
RNU6 & CTCGCTTCGGCAGCACA & AACGCTTCACGAATTGCGT \\
swoyry & TTCCTGGATGAGTGTCCTGGG & TATGGTGAGGGCAGTTGTCTCT \\
TC0100011037 & TTGAGTTAGCGAGTGGGGAGA & TGCAAATCTGGGGTTTGACCT \\
TC0100013007 & GGAAAGTCTCTGAGGAAACAGCA & GAGTAACCCATGCCTGCTCC \\
TC0100015528 & CACCTAGCCATCCCCACTGA & TTCTTTTGCTTGTGGCGTGC \\
TC0100016418 & TGACACAGGATAAGCGCAACA & CCCCCTTTACCTTCCTTGAGC \\
TC0400008478 & GCTCTGGAAAACCACAGGGTC & ATAGATCTGTGGCCAGGTGAGG \\
TC0600007784 & CCTGATCCATGCTAGAGGTTGA & TGGAGAAACTCAATGACACCAGAAG \\
TC1400008125 & AGTTGCAAGAACGAACGGGA & CATAGGCTGGCTTGTGGAGG \\
TC1600007870 & CGCCTCTACCTCCAGTGTGA & GGCCAGAGTGGAGCCATGTA \\
TC1900010363 & AGGAGGAGACACACCCAAAAGA & GAATGCTTTTTAAGGGTGCGAGC \\
UBE2 21 & GTTGTCCTGCAAGAGCTTCG & TGTAACACTGTCCTTCGGGC \\
zyjeebu & TGTTGGCACAGTCCGTTGTC & CTCCCCTAACCTCACAGGCA \\
\hline
\end{tabular}

PCR reactions were performed using 50 ng RNA in input for each assay and Power SYBR ${ }^{\circledR}$ Green RNA-to-CT ${ }^{\mathrm{TM}}$ 1-Step Kit (Thermo Fisher Scientific, Waltham, MA, USA), according to the manufacturer's instructions. GAPDH (glyceraldehyde-3-phosphate dehydrogenase) and RNU6 (RNA, U6 small nuclear 1) were used as endogenous controls. All reactions were performed on a 7900HT Fast Real-Time PCR System (Foster City, CA, USA). DE transcripts were identified using SDS RQ Manager 1.2 software (Foster City, CA, USA); by applying the $2^{-\Delta \Delta C t}$ method, and differential expression was expressed as $R Q$ (Relative Quantity); $R Q$ values $<1$ were converted in fold change (FC) by applying the formula $-1 / R Q$. 


\subsection{ROC Curve Analysis}

To assess the potential diagnostic accuracy of DE transcripts, we computed ROC curves using SPSS 23 (IBM). For each ROC curve, generated by using expression values ( $\Delta C \mathrm{ts}$ ), area under the curve (AUC), 95\% confidence intervals (CIs) and $p$-value were calculated; the Youden method was applied to identify the optimal cut-off, with the associated sensitivity, specificity, accuracy, positive predictive value (PPV) and negative predictive values (NPV). In order to investigate if different combinations of DE transcripts could increase diagnostic accuracy, we also computed a ROC curve generated from a molecular signature of multiple transcripts: this multivariable ROC curve was computed considering all DE transcripts by building a binary logistic regression model through SPSS [60]. Statistical significance was established at a $p$-value $<0.05$ for all ROC curves.

\subsection{Expression Analysis in Peripheral Blood Mononuclear Cells}

Expression of DE transcripts was also investigated in cells isolated from peripheral blood of 10 $\mathrm{AD}$ patients and 10 non-matched unaffected individuals included in the validation cohort. Peripheral blood mononuclear cells (PBMCs) from patients and controls were isolated from heparinized whole blood by gradient centrifugation over Ficoll-Hypaque solution (Ficoll Paque PLUS-GE Healthcare Life Sciences, Piscataway, NJ, USA). Lymphocytes were prepared from blood samples by using the Lympholyte ${ }^{\circledR}-\mathrm{H}$ density gradient separation medium (Cedarlane, Burlington, NC, USA) according to the manufacturer's instructions, with slight modifications. Briefly, the blood was diluted by adding an equal volume of complete RPMI 1640 medium ( $20 \%$ FBS, $1 \%$ penicillin/streptomycin, $1 \%$ L-glutammine, and $5 \%$ Phytohaemagglutinin). Two parts of diluted blood were added to one part of Lympholyte ${ }^{\circledR}-\mathrm{H}$. A centrifugation step ( $400 \times g$ for $25 \mathrm{~min}$ ) was performed to separate the lymphocyte fraction from the whole blood; a well-defined lymphocyte layer appeared at the interface at the end of this step. The lymphocyte fraction was removed from the interface and transferred to a new centrifuge tube containing complete RPMI 1640 medium, followed by a centrifugation step (400 $\mathrm{g}$ for $10 \mathrm{~min}$ ). After two additional washing steps, the isolated lymphocyte fraction was incubated in $25 \mathrm{~cm}^{2}$ culture flasks containing RPMI medium for $72 \mathrm{~h}$ at $37^{\circ} \mathrm{C}$ and $5 \% \mathrm{CO}_{2}$. Lymphocytes $\left(4 \times 10^{6}\right)$ were then stored at $-80{ }^{\circ} \mathrm{C}$ until use. Total RNA was isolated from PBMC pellets by using TRIzol (Thermo Fisher Scientific, Waltham, MA, USA), according to the manufacturer's instructions. Expression analysis was performed by Real-Time PCR as described above, using 50 ng of RNA for each assay. ACTB (actin beta) and RNU6 were used as endogenous controls.

\subsection{Statistical Analysis}

Statistical analysis of expression data was performed using GraphPad Prism 8. PCR data $(\Delta C t s)$ were tested for normality of distributions ( $\mathrm{D}^{\prime}$ Agostino \& Pearson omnibus normality test and Shapiro-Wilk normality test) and homogeneity of variance (F test); according to the results, parametric or non-parametric $t$-test was applied to evaluate statistically significant differences in transcript expression. Both paired (paired $t$-test or Wilcoxon test) and unpaired (homoscedastic or Welch corrected unpaired $t$-test, or Mann-Whitney test) analysis were performed.

To assess if age and sex represented confounding factors in this study, we followed a three-step procedure [61]: (1) the difference between $\mathrm{AD}$ patients and unaffected individuals in age and sex was evaluated through $t$-test/Mann-Whitney test (according to normality of distributions); (2) the association of sex/age with disease risk was evaluated using Binomial Logistic Regression; (3) the association of sex/age with DE transcript expression was evaluated by calculating Pearson/Spearman correlation coefficient (according to normality of distributions).

Correlation analysis was performed to evaluate the existence of relationships between plasma expression levels of DE transcripts $(-\Delta C \mathrm{ts})$ and cognitive decline of $\mathrm{AD}$ patients and unaffected individuals. After checking for normality of distributions, Pearson/Spearman correlation coefficient was calculated. Correlation $p$-values were corrected for multiple comparisons (Holm-Sidak method). 


\section{Conclusions}

This study identified six transcripts (GS1-304P7.3, NONHSAT090268, TC0100011037, TC0400008478, TC1400008125, and UBE2V1) showing an altered expression in the plasma of mild-moderate $\mathrm{AD}$ patients compared to unaffected individuals. Given the need of new accurate blood biomarkers for $\mathrm{AD}$ diagnosis, these transcripts may be considered for further analyses in larger cohorts, also in combination with other biomarkers (including both $\mathrm{A} \beta$-based biomarkers and miRNAs or other RNA molecules), with the aim of identifying specific RNA-based biomarkers to be eventually validated and introduced into clinical practice. Further studies enrolling patients with mild cognitive impairment would be useful to investigate if DE transcript expression may predict the severity of cognitive decline, potentially in an early phase of $\mathrm{AD}$ pathogenesis.

Author Contributions: M.R., M.P., F.C., and R.F. designed and conceived the experiments. M.G., M.G.S., F.I.I.C., and G.C. obtained and characterized biological samples from patients. C.B., M.T.D.M., and F.S. performed the experiments. C.B. and M.R. contributed to the analysis and interpretation of data. C.B., M.R., M.T.D.M., and F.C. wrote the paper; D.B. and C.D.P. reviewed and edited the manuscript. All authors have read and agreed to the published version of the manuscript.

Funding: This research received no external funding.

Acknowledgments: The authors wish to thank the Scientific Bureau of the University of Catania for language support.

Conflicts of Interest: The authors declare no conflict of interest.

\section{Abbreviations}

\section{$\mathrm{AD}$}

ADAMTS9

ADAMTS9-AS2

ARSJ

AUC

$\mathrm{A} \beta$

circRNAs

CIs

CNS

CSF

CTRL

DE

GAPDH

IncRNAs

miRNAs

MMSE

ncRNAs

NPV

PBMCs

PDC

PDC-AS1

PPV

PSD95

PTGS2

RNU6

ROC

RPL23A

UBE2V1

UGT8

\author{
Alzheimer's disease \\ ADAM metallopeptidase with thrombospondin type 1 motif 9 \\ ADAMTS9 antisense RNA 2 \\ arylsulfatase family member J \\ area under the curve \\ amyloid beta \\ circular RNAs \\ confidence intervals \\ central nervous system \\ cerebrospinal fluid \\ Controls \\ differentially expressed \\ glyceraldehyde-3-phosphate dehydrogenase \\ long non-coding RNAs \\ microRNAs \\ Mini Mental State Examination \\ non-coding RNAs \\ negative predictive value \\ peripheral blood mononuclear cells \\ Phosducin \\ PDC antisense RNA 1 \\ positive predictive value \\ postsynaptic density-95 \\ prostaglandin-endoperoxide synthase 2 \\ RNA, U6 small nuclear 1 \\ receiver operating characteristic \\ ribosomal protein L23a \\ ubiquitin conjugating enzyme E2 V1) \\ UDP glycosyltransferase 8
}




\section{References}

1. Khoury, R.; Grossberg, G.T. Deciphering Alzheimer's disease: Predicting new therapeutic strategies via improved understanding of biology and pathogenesis. Expert Opin. Ther. Targets 2020, 24, 859-868. [CrossRef] [PubMed]

2. Caraci, F.; Castellano, S.; Salomone, S.; Drago, F.; Bosco, P.; Nuovo, S. Searching for Disease-Modifying Drugs in AD: Can We Combine Neuropsychological Tools with Biological Markers? CNS Neurol. Disord. Drug Targets 2014, 13, 173-186. [CrossRef] [PubMed]

3. Caraci, F.; Copani, A.; Nicoletti, F.; Drago, F. Depression and Alzheimer's disease: Neurobiological links and common pharmacological targets. Eur. J. Pharmacol. 2010, 626, 64-71. [CrossRef] [PubMed]

4. Jack, C.R.; Bennett, D.A.; Blennow, K.; Carrillo, M.C.; Dunn, B.; Haeberlein, S.B.; Holtzman, D.M.; Jagust, W.; Jessen, F.; Karlawish, J.; et al. NIA-AA Research Framework: Toward a biological definition of Alzheimer's disease. Alzheimer's Dement. 2018, 14, 535-562. [CrossRef]

5. Sharma, N. Exploring Biomarkers for Alzheimer's Disease. J. Clin. Diagn. Res. 2016, 10, KE01-KE06. [CrossRef]

6. Hampel, H.; Vergallo, A.; Caraci, F.; Cuello, A.C.; Lemercier, P.; Vellas, B.; Giudici, K.V.; Baldacci, F.; Hänisch, B.; Haberkamp, M.; et al. Future avenues for Alzheimer's disease detection and therapy: Liquid biopsy, intracellular signaling modulation, systems pharmacology drug discovery. Neuropharmacology 2020, 108081. [CrossRef]

7. Zetterberg, H.; Burnham, S.C. Blood-based molecular biomarkers for Alzheimer's disease. Mol. Brain 2019, 12, 26. [CrossRef]

8. Barbagallo, C.; Mostile, G.; Baglieri, G.; Giunta, F.; Luca, A.; Raciti, L.; Zappia, M.; Purrello, M.; Ragusa, M.; Nicoletti, A. Specific Signatures of Serum miRNAs as Potential Biomarkers to Discriminate Clinically Similar Neurodegenerative and Vascular-Related Diseases. Cell. Mol. Neurobiol. 2019, 40, 531-546. [CrossRef]

9. Sheinerman, K.S.; Toledo, J.B.; Tsivinsky, V.G.; Irwin, D.; Grossman, M.; Weintraub, D.; Hurtig, H.I.; Chen-Plotkin, A.; Wolk, D.A.; McCluskey, L.F.; et al. Circulating brain-enriched microRNAs as novel biomarkers for detection and differentiation of neurodegenerative diseases. Alzheimer's Res. Ther. 2017, 9, 89. [CrossRef]

10. Cirnigliaro, M.; Barbagallo, C.; Gulisano, M.; Domini, C.N.; Barone, R.; Barbagallo, D.; Ragusa, M.; Di Pietro, C.; Rizzo, R.; Purrello, M. Expression and Regulatory Network Analysis of miR-140-3p, a New Potential Serum Biomarker for Autism Spectrum Disorder. Front. Mol. Neurosci. 2017, 10, 250. [CrossRef]

11. Di Pietro, V.; Porto, E.; Ragusa, M.; Barbagallo, C.; Davies, D.; Forcione, M.; Logan, A.; Di Pietro, C.; Purrello, M.; Grey, M.; et al. Salivary MicroRNAs: Diagnostic Markers of Mild Traumatic Brain Injury in Contact-Sport. Front. Mol. Neurosci. 2018, 11, 290. [CrossRef] [PubMed]

12. Filipów, S.; Łaczmański, Ł. Blood Circulating miRNAs as Cancer Biomarkers for Diagnosis and Surgical Treatment Response. Front. Genet. 2019, 10, 169. [CrossRef] [PubMed]

13. Drokow, E.K.; Sun, K.; Ahmed, H.A.W.; Akpabla, G.S.; Song, J.; Shi, M. Circulating microRNA as diagnostic biomarkers for haematological cancers: A systematic review and meta-analysis. Cancer Manag. Res. 2019, 11, 4313-4326. [CrossRef] [PubMed]

14. Di Mauro, S.; Ragusa, M.; Urbano, F.; Filippello, A.; Di Pino, A.; Scamporrino, A.; Pulvirenti, A.; Ferro, A.; Rabuazzo, A.M.; Purrello, M.; et al. Intracellular and extracellular miRNome deregulation in cellular models of NAFLD or NASH: Clinical implications. Nutr. Metab. Cardiovasc. Dis. 2016, 26, 1129-1139. [CrossRef]

15. Párrizas, M.; Novials, A. Circulating microRNAs as biomarkers for metabolic disease. Best Pract. Res. Clin. Endocrinol. Metab. 2016, 30, 591-601. [CrossRef]

16. Battaglia, R.; Palini, S.; Vento, M.E.; La Ferlita, A.; Lo Faro, M.J.; Caroppo, E.; Borzì, P.; Falzone, L.; Barbagallo, D.; Ragusa, M.; et al. Identification of extracellular vesicles and characterization of miRNA expression profiles in human blastocoel fluid. Sci. Rep. 2019, 9, 84. [CrossRef]

17. Alsaweed, M.; Hartmann, P.; Geddes, D.; Kakulas, F. MicroRNAs in Breastmilk and the Lactating Breast: Potential Immunoprotectors and Developmental Regulators for the Infant and the Mother. Int. J. Environ. Res. Public Health 2015, 12, 13981-14020. [CrossRef]

18. Layne, T.R.; Green, R.A.; Lewis, C.A.; Nogales, F.; Cruz, T.C.D.; Zehner, Z.E.; Seashols-Williams, S.J. MicroRNA Detection in Blood, Urine, Semen, and Saliva Stains After Compromising Treatments. J. Forensic Sci. 2019, 64, 1831-1837. [CrossRef] 
19. Kim, Y.-K.; Song, J. The Role of Long Noncoding RNAs in Diabetic Alzheimer's Disease. J. Clin. Med. 2018, 7, 461. [CrossRef]

20. Hampel, H.; Caraci, F.; Cuello, A.C.; Caruso, G.; Nisticò, R.; Corbo, M.; Baldacci, F.; Toschi, N.; Garaci, F.; Chiesa, P.A.; et al. A Path Toward Precision Medicine for Neuroinflammatory Mechanisms in Alzheimer's Disease. Front. Immunol. 2020, 11, 456. [CrossRef]

21. Itzhaki, R.F.; Lathe, R.; Balin, B.J.; Ball, M.J.; Bearer, E.L.; Braak, H.; Bullido, M.J.; Carter, C.; Clerici, M.; Cosby, S.L.; et al. Microbes and Alzheimer's Disease. J. Alzheimer's Dis. 2016, 51, 979-984. [CrossRef] [PubMed]

22. Bialopiotrowicz, E.; Kuzniewska, B.; Kachamakova-Trojanowska, N.; Barcikowska, M.; Kuznicki, J.; Wojda, U. Cell cycle regulation distinguishes lymphocytes from sporadic and familial Alzheimer's disease patients. Neurobiol. Aging 2011, 32, 2319.e13-2319.e26. [CrossRef]

23. Rodrigues, R.; Debom, G.; Soares, F.; Machado, C.; Pureza, J.; Peres, W.; Garcias, G.d.L.; Duarte, M.F.; Schetinger, M.R.C.; Stefanello, F.; et al. Alterations of ectonucleotidases and acetylcholinesterase activities in lymphocytes of Down syndrome subjects: Relation with inflammatory parameters. Clin. Chim. Acta 2014, 433, 105-110. [CrossRef] [PubMed]

24. Cuello, A.C. Early and Late CNS Inflammation in Alzheimer's Disease: Two Extremes of a Continuum? Trends Pharmacol. Sci. 2017, 38, 956-966. [CrossRef] [PubMed]

25. Xu, N.; Gulick, J.; Osinska, H.; Yu, Y.; McLendon, P.M.; Shay-Winkler, K.; Robbins, J.; Yutzey, K.E. Ube2v1 Positively Regulates Protein Aggregation by Modulating Ubiquitin Proteasome System Performance Partially Through K63 Ubiquitination. Circ. Res. 2020, 126, 907-922. [CrossRef]

26. Sanfeliu, A.; Hokamp, K.; Gill, M.; Tropea, D. Transcriptomic Analysis of Mecp2 Mutant Mice Reveals Differentially Expressed Genes and Altered Mechanisms in Both Blood and Brain. Front. Psychiatry 2019, 10, 278. [CrossRef]

27. Ma, Q.; Ruan, H.; Peng, L.; Zhang, M.; Gack, M.U.; Yao, W.-D. Proteasome-independent polyubiquitin linkage regulates synapse scaffolding, efficacy, and plasticity. Proc. Natl. Acad. Sci. USA 2017, 114, E8760-E8769. [CrossRef]

28. Hou, Y.; Zhang, R.; Sun, X. Enhancer LncRNAs Influence Chromatin Interactions in Different Ways. Front. Genet. 2019, 10, 936. [CrossRef]

29. Romero-Barrios, N.; Legascue, M.F.; Benhamed, M.; Ariel, F.; Crespi, M. Splicing regulation by long noncoding RNAs. Nucleic Acids Res. 2018, 46, 2169-2184. [CrossRef]

30. Müller, R.; Weirick, T.; John, D.; Militello, G.; Chen, W.; Dimmeler, S.; Uchida, S. ANGIOGENES: Knowledge database for protein-coding and noncoding RNA genes in endothelial cells. Sci. Rep. 2016, 6, 32475. [CrossRef]

31. Vagnucci, A.H.; Li, W.W. Alzheimer's disease and angiogenesis. Lancet 2003, 361, 605-608. [CrossRef]

32. Castro, M.G.; Biron, K.E.; Dickstein, D.L.; Gopaul, R.; Jefferies, W.A. Amyloid Triggers Extensive Cerebral Angiogenesis Causing Blood Brain Barrier Permeability and Hypervascularity in Alzheimer's Disease. PLoS ONE 2011, 6, e23789. [CrossRef]

33. Ragusa, M.; Barbagallo, C.; Brex, D.; Caponnetto, A.; Cirnigliaro, M.; Battaglia, R.; Barbagallo, D.; Di Pietro, C.; Purrello, M. Molecular Crosstalking among Noncoding RNAs: A New Network Layer of Genome Regulation in Cancer. Int. J. Genom. 2017, 2017, 1-17. [CrossRef] [PubMed]

34. Broeckel, U.; Stoll, M.; Hein, L. The identification of phosducin as a novel candidate gene for hypertension and its role in sympathetic activation. Curr. Opin. Nephrol. Hypertens. 2011, 20, 118-124. [CrossRef] [PubMed]

35. Beetz, N.; Harrison, M.D.; Brede, M.; Zong, X.; Urbanski, M.J.; Sietmann, A.; Kaufling, J.; Barrot, M.; Seeliger, M.W.; Vieira-Coelho, M.A.; et al. Phosducin influences sympathetic activity and prevents stress-induced hypertension in humans and mice. J. Clin. Investig. 2009, 119, 3597-3612. [CrossRef]

36. Skoog, I.; Gustafson, D. Update on hypertension and Alzheimer's disease. Neurol. Res. 2013, $28,605-611$. [CrossRef]

37. García-Alberca, J.M.; Mendoza, S.; Gris, E.; Royo, J.L.; Cruz-Gamero, J.M.; García-Casares, N. White matter lesions and temporal atrophy are associated with cognitive and neuropsychiatric symptoms in patients with hypertension and Alzheimer's disease. Int. J. Geriatr. Psychiatry 2020. [CrossRef] 
38. Chen, Q.; Liang, B.; Wang, Z.; Cheng, X.; Huang, Y.; Liu, Y.; Huang, Z. Influence of four polymorphisms in ABCA1 and PTGS2 genes on risk of Alzheimer's disease: A meta-analysis. Neurol. Sci. 2016, 37, 1209-1220. [CrossRef]

39. Ma, S.L.; Tang, N.L.S.; Zhang, Y.P.; Ji, L.-d.; Tam, C.W.C.; Lui, V.W.C.; Chiu, H.F.K.; Lam, L.C.W. Association of prostaglandin-endoperoxide synthase 2 (PTGS2) polymorphisms and Alzheimer's disease in Chinese. Neurobiol. Aging 2008, 29, 856-860. [CrossRef]

40. Wang, P.; Guan, P.-P.; Wang, T.; Yu, X.; Guo, J.-J.; Wang, Z.-Y. Aggravation of Alzheimer's disease due to the COX-2-mediated reciprocal regulation of IL-1 $\beta$ and A $\beta$ between glial and neuron cells. Aging Cell 2014, 13, 605-615. [CrossRef]

41. Woodling, N.S.; Colas, D.; Wang, Q.; Minhas, P.; Panchal, M.; Liang, X.; Mhatre, S.D.; Brown, H.; Ko, N.; Zagol-Ikapitte, I.; et al. Cyclooxygenase inhibition targets neurons to prevent early behavioural decline in Alzheimer's disease model mice. Brain 2016, 139, 2063-2081. [CrossRef] [PubMed]

42. Garcia-Esparcia, P.; Sideris-Lampretsas, G.; Hernandez-Ortega, K.; Grau-Rivera, O.; Sklaviadis, T.; Gelpi, E.; Ferrer, I. Altered mechanisms of protein synthesis in frontal cortex in Alzheimer disease and a mouse model. Am. J. Neurodegener. Dis. 2017, 6, 15-25. [PubMed]

43. Evans, H.T.; Benetatos, J.; van Roijen, M.; Bodea, L.G.; Götz, J. Decreased synthesis of ribosomal proteins in tauopathy revealed by non-canonical amino acid labelling. EMBO J. 2019, 38, e101174. [CrossRef] [PubMed]

44. Cooper-Knock, J.; Shaw, P.J.; Moll, T. Disrupted glycosylation of lipids and proteins is a cause of neurodegeneration. Brain 2020, 143, 1332-1340. [CrossRef]

45. McKenzie, A.T.; Moyon, S.; Wang, M.; Katsyv, I.; Song, W.-M.; Zhou, X.; Dammer, E.B.; Duong, D.M.; Aaker, J.; Zhao, Y.; et al. Multiscale network modeling of oligodendrocytes reveals molecular components of myelin dysregulation in Alzheimer's disease. Mol. Neurodegener. 2017, 12, 82. [CrossRef]

46. Katsel, P.; Li, C.; Haroutunian, V. Gene Expression Alterations in the Sphingolipid Metabolism Pathways during Progression of Dementia and Alzheimer's Disease: A Shift Toward Ceramide Accumulation at the Earliest Recognizable Stages of Alzheimer's Disease? Neurochem. Res. 2007, 32, 845-856. [CrossRef]

47. Yao, J.; Zhou, B.; Zhang, J.; Geng, P.; Liu, K.; Zhu, Y.; Zhu, W. A new tumor suppressor LncRNA ADAMTS9-AS2 is regulated by DNMT1 and inhibits migration of glioma cells. Tumor Biol. 2014, 35, 7935-7944. [CrossRef]

48. Kamboh, M.I.; Demirci, F.Y.; Wang, X.; Minster, R.L.; Carrasquillo, M.M.; Pankratz, V.S.; Younkin, S.G.; Saykin, A.J.; Jun, G.; Baldwin, C.; et al. Genome-wide association study of Alzheimer's disease. Transl. Psychiatry 2012, 2, e117. [CrossRef]

49. Yao, Y.-G.; Lin, E.; Tsai, S.-J.; Kuo, P.-H.; Liu, Y.-L.; Yang, A.C.; Kao, C.-F.; Yang, C.-H. The ADAMTS9 gene is associated with cognitive aging in the elderly in a Taiwanese population. PLoS ONE 2017, 12, e0172440. [CrossRef]

50. Li, N.; Li, J.; Mi, Q.; Xie, Y.; Li, P.; Wang, L.; Binang, H.; Wang, Q.; Wang, Y.; Chen, Y.; et al. Long non-coding RNA ADAMTS9-AS1 suppresses colorectal cancer by inhibiting the Wnt/ $\beta$-catenin signalling pathway and is a potential diagnostic biomarker. J. Cell. Mol. Med. 2020. [CrossRef]

51. Caricasole, A. Induction of Dickkopf-1, a Negative Modulator of the Wnt Pathway, Is Associated with Neuronal Degeneration in Alzheimer's Brain. J. Neurosci. 2004, 24, 6021-6027. [CrossRef] [PubMed]

52. Inestrosa, N.; Tapia-Rojas, C. Loss of canonical Wnt signaling is involved in the pathogenesis of Alzheimer's disease. Neural Regen. Res. 2018, 13, 1705. [CrossRef] [PubMed]

53. Khoury, R.; Ghossoub, E. Diagnostic biomarkers of Alzheimer's disease: A state-of-the-art review. Biomark. Neuropsychiatry 2019, 1, 100005. [CrossRef]

54. Risacher, S.L.; Fandos, N.; Romero, J.; Sherriff, I.; Pesini, P.; Saykin, A.J.; Apostolova, L.G.; Zetterberg, H. Plasma amyloid beta levels are associated with cerebral amyloid and tau deposition. Alzheimer's Dement. Diagn. Assess. Dis. Monit. 2019, 11, 510-519. [CrossRef] [PubMed]

55. Nakamura, A.; Kaneko, N.; Villemagne, V.L.; Kato, T.; Doecke, J.; Doré, V.; Fowler, C.; Li, Q.-X.; Martins, R.; Rowe, C.; et al. High performance plasma amyloid- $\beta$ biomarkers for Alzheimer's disease. Nature 2018, 554, 249-254. [CrossRef]

56. McKhann, G.; Drachman, D.; Folstein, M.; Katzman, R.; Price, D.; Stadlan, E.M. Clinical diagnosis of Alzheimer's disease: Report of the NINCDS-ADRDA Work Group* under the auspices of Department of Health and Human Services Task Force on Alzheimer's Disease. Neurology 1984, 34, 939-944. [CrossRef]

57. Folstein, M.F.; Folstein, S.E.; McHugh, P.R. Mini-mental state. J. Psychiatr. Res. 1975, 12, 189-198. [CrossRef] 
58. Doody, R.S.; Massman, P.; Dunn, J.K. A Method for Estimating Progression Rates in Alzheimer Disease. Arch. Neurol. 2001, 58, 449-454. [CrossRef]

59. Di Mauro, S.; Scamporrino, A.; Petta, S.; Urbano, F.; Filippello, A.; Ragusa, M.; Di Martino, M.T.; Scionti, F.; Grimaudo, S.; Pipitone, R.M.; et al. Serum coding and non-coding RNAs as biomarkers of NAFLD and fibrosis severity. Liver Int. 2019, 39, 1742-1754. [CrossRef]

60. Chan, Y.H. Biostatistics 202: Logistic regression analysis. Singap. Med. J. 2004, 45, 149-153.

61. Jager, K.J.; Zoccali, C.; MacLeod, A.; Dekker, F.W. Confounding: What it is and how to deal with it. Kidney Int. 2008, 73, 256-260. [CrossRef] [PubMed]

Publisher's Note: MDPI stays neutral with regard to jurisdictional claims in published maps and institutional affiliations.

(C) 2020 by the authors. Licensee MDPI, Basel, Switzerland. This article is an open access article distributed under the terms and conditions of the Creative Commons Attribution (CC BY) license (http://creativecommons.org/licenses/by/4.0/). 